\title{
AICSP NorCAS 2020 S.I. Foreword
}

\author{
Kristian G. Kjelgård ${ }^{1} \cdot$ Dag T. Wisland ${ }^{1}$
}

Published online: 10 January 2022

(C) The Author(s), under exclusive licence to Springer Science+Business Media, LLC, part of Springer Nature 2021

This special issue includes extended versions of selected papers presented at the 2020 6th NorCAS conference. The 6th edition was planned to be hosted in Oslo, Norway, but due travel restrictions in the COVID-19 pandemic, the conference was hosted as a virtual event. NorCAS is a regional annual conference for the circuits and system community located in the Nordic and Baltic countries. From a total of 58 submitted paper, 29 was selected for presentation. Of the 13 papers in the Analog Track three selected papers was extended and accepted for journal publication in this special issue.

Paper 1: A Configurable Fluorescence Sensing Frontend for Ultra-Low Power and High Sensitivity Applications This paper presents a configurable fluorescence sensing font-end for implantable cell level measurements. The font-end includes three photo-diode implementations and three transimpedance implementations accommodating a wide photo current range of $1 \mathrm{pA}$ to $10 \mathrm{~mA}$. The design also features an ambient light cancellations technique and is implemented in 0.35 um standard CMOS. In the ultralow-power configuration the power consumption is $119 \mathrm{nW}$ with an integrated noise of $210 \mathrm{pA}$ at $20 \mathrm{kHz}$.

Paper 2: Schmitt Trigger Based Single-Ended Voltage Amplifier for Ultra-Low-Voltage Supplies In this paper a a Schmitt Trigger based pseudo-differential single-ended voltage amplifier is presented. By using a Schmitt Trigger in parallel with a conventional inverter, hysteresis is eliminated and voltage gain is improved. The measured DC voltage gain at $0.2 \mathrm{~V}$ supply voltage is $24 \mathrm{~dB}$. The simulated unity-gain-bandwidth is $434 \mathrm{kHz}$, giving a 90 degree phase margin with a $10 \mathrm{pF}$ capacitive load. The power consumption is $488 \mathrm{nW}$.

Paper 3: Origins and Minimization of Intermodulation Distortion in A Pseudo-differential CMOS Beamforming Receiver The third paper studies how nonlinear distortion

\footnotetext{
Kristian G. Kjelgård

kristigk@ifi.uio.no

1 Oslo, Norway
}

is generated in an inverter-based front-end with passive mixer. By utilizing pseudo-differential common-mode feedback, the second order intermodulation is cancelled. In addition, the third order intermodulation caused by cascaded secondary order non-linearities is reduced significantly. The paper discusses spurious tones rising in the mixing, and how to extend the analysis into actual frequency translation effect.

Finally, we would thank the authors, reviewers and editors dedicating their efforts to this publication.

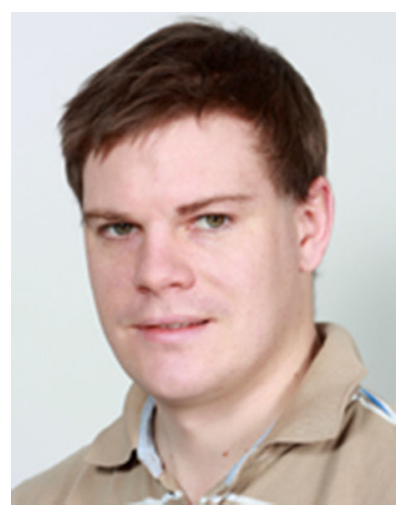

Kristian G. Kjelgård received his M.Sc. and Ph.D. in Microelectronics from the University Oslo, in 2010 and 2015, respectively. He is currently an Associate Professor of microelectronics and microwave systems at the Department of Informatics, University of Oslo, Norway. His current research interests include microwave integrated circuits, biomedical sensors and ultra-wideband radar systems. He in a senior member of IEEE and from 2020 he is the Technical Program Committee Analog Chair of the IEEE NorCAS conference.

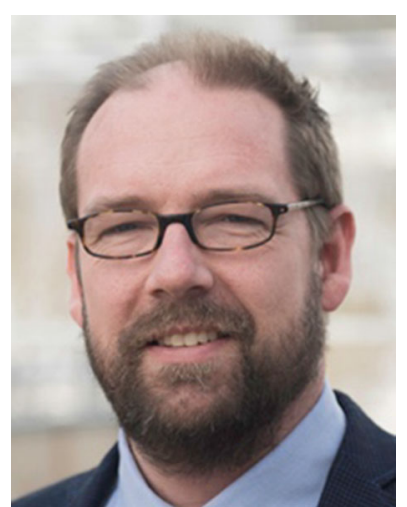

Dag T. Wisland received the M.Sc. and Dr. Scient. degrees in electrical engineering from the University of Oslo, Oslo, Norway, in 1996 and 2003, respectively. He is a Professor and Head of research of the Micro-l Nanoelectronics Group, University of Oslo. He is a cofounder of the semiconductor company Novelda AS and was the CEO from 2004 to 2013 when he was appointed CTO, a position he still holds. Wisland has been strongly involved in bringing innovative products to the market, both consumer and 
industrial. He has authored or co-authored several scientific publications and been the principal investigator/project manager for large number of international research and innovation projects. Dr. Wisland is a senior member of IEEE and serves as a TC member of the IEEE CAS society Analog Signal Processing (ASP) and Biomedical Circuits and Systems (BIOCAS) technical committees. He has also been involved as an RCM for several ISCAS conferences. From 2020 he is a member of the IEEE CAS Industrial Pioneer Award Committee. Wisland contributed to founding the UWB Alliance and serves as a BoD member. He is also a member of the ETSI TG-UWB group and has contributed to several ETSI standards. 